\title{
Point Defects in Blue Phosphorene
}

\author{
Minglei Sun†, Jyh-Pin Chou ${ }^{\ddagger}$, Alice $\mathrm{Hu}^{\ddagger}$, and Udo Schwingenschlögl†,* \\ †Physical Science and Engineering Division (PSE), King Abdullah University of Science and Technology (KAUST), \\ Thuwal 23955-6900, Saudi Arabia \\ ‡Department of Mechanical and Biomedical Engineering, City University of Hong Kong, Kowloon 999077, China \\ *udo.schwingenschlogl@kaust.edu.sa
}

\begin{abstract}
Using first-principles calculations, we investigate selected defects in blue phosphorene (BlueP). For a single vacancy (SV) defect a 5-9 structure is energetically favorable and for a double vacancy (DV) defect a 5-8-5 or 555-777 structure. A P adatom favors the top adsorption site. Scanning tunneling microscopy images are simulated to aid the experimental identification of the defects. Formation of a Stone-Wales defect is found to be most likely, but can be reverted by thermal annealing. Calculated migration and transformation barriers show that a SV defect can migrate easily. Both a SV defect and a $\mathrm{P}$ adatom induce a magnetic moment, thus turning BlueP into a magnetic semiconductor. It turns out that all the defects under investigation enhance the ability of BlueP to absorb sunlight.
\end{abstract}

\section{INTRODUCTION}

The fascinating properties of two-dimensional (2D) materials endow them with potential to be used for numerous purposes. Applications in nanoelectronic devices require a sizeable bandgap, which is provided, for example, by blue phosphorene (BlueP), $\underline{1}$ with a carrier mobility in excess of $10^{3} \mathrm{~cm}^{2} \cdot \mathrm{V}^{-1} \cdot \mathrm{s}^{-1}$. 2 The effects of doping, $\underline{3-5}$ functionalization, $\frac{6-9}{1}$ and heterostructure formation,,$\frac{10-15}{15}$ including external stimuli such as strain and electric field, on the electronic and magnetic properties of BlueP are known from first-principles calculations, indicating that it is a promising 2D material. Various investigations have explored the potential in nanoelectronic devices,,$\underline{16}$ photodetectors,, 17 energy storage, $, \underline{15}, \underline{18-21}$ gas sensors, $\underline{22-24}$ and superconductors. $\underline{25}$ While BlueP can be grown by molecular beam epitaxy on the $\mathrm{Au}(111)$ surface, $\underline{26,27}$ defects are inevitable during synthesis of 2D materials. $\underline{28-32}$ On the other hand, defects may be generated artificially by ion or electron irradiation $\frac{33-36}{3}$ to cultivate novel properties and expand the range of applications.

First, defects can be introduced to tune the magnetic properties of 2D materials. For example, graphene is of interest in spintronics owing to its long spin-relaxation length and spin lifetime. $\frac{37}{7}$ While pristine graphene is non-magnetic, magnetism can be triggered by simple defects, such as a single vacancy (SV) due to the created dangling bond, and remains stable over a wide range of defect concentrations. $\frac{38-40}{}$ Following predictions that adatoms can induce magnetism in graphene, 11,42 it has been demonstrated experimentally that both $\mathrm{F}$ adatoms and irradiation defects lead to paramagnetism. $\underline{43}$ Defects can also tune the magnetic properties of other 2D materials such as silicene, $\underline{44}$ $\mathrm{MoS}_{2}, \underline{45-47}$ and phosphorene. $\frac{48}{}$ Second, defects can be used to tune the electronic properties of $2 \mathrm{D}$ materials. In the case of 2D Dirac materials the bandgap needed for devices such as field-effect transistors can be opened by introducing a defect that breaks the intrinsic symmetry. For example, a Stone-Wales (SW) defect in silicene opens a direct bandgap of $33 \mathrm{meV}$, almost without changing the linear band dispersions, giving rise to a semiconductor with high carrier mobility. $\underline{44}$ On the other hand, the bandgaps of the 2D semiconductors arsenene, antimonene, and antimony arsenide can be reduced significantly by defects. 49,50 Third, defects modify optical properties, for example, in the cases

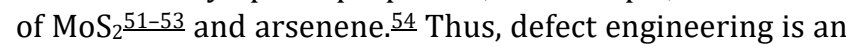
effective way to tailor 2D materials according to technological needs and it is of great interest to explore defects in BlueP.

In this paper, we systematically investigate SW, SV, double vacancy (DV), and P adatom defects in BlueP. We begin by obtaining the favorable defect structures and providing guidance to experiment by simulating their scanning tunneling microscopy (STM) images. Formation, migration, and transformation of the defects are studied, and their effects on the electronic, magnetic, and optical properties of BlueP are examined in detail.

\section{COMPUTATIONAL DETAILS}

First-principles calculations are carried out using the Vienna Ab-initio Simulation Package (projector-augmented wave method) $\underline{55}$ with $400 \mathrm{eV}$ energy cutoff of the planewave basis. The Perdew-Burke-Ernzerhof functional $\frac{56}{6}$ is employed for structure relaxation and, as this functional typically underestimates the bandgap of semiconductors, the Heyd-Scuseria-Ernzerhof hybrid functional 57 for calculating electronic and optical properties. Spin-polarization is taken into account in all calculations. BlueP with defects is modeled in $5 \times 5 \times 1$ supercells with periodic boundary conditions, because for $7 \times 7 \times 1$ supercells virtually the same electronic and optical properties are obtained. Empty 
space of at least $15 \AA$ thickness is inserted to avoid interaction between periodic images of the $2 \mathrm{D}$ material. The Brillouin zone is sampled on a $6 \times 6 \times 1 \Gamma$-centered MonkhorstPack $k$-grid (structure relaxation: $2 \times 2 \times 1$ ). Each structure is relaxed until all Hellmann-Feynman forces remain below $0.01 \mathrm{~V} \cdot \AA^{-1}$. The climbing-image nudged-elastic-band method $\underline{58}$ is used to calculate the transformation and migration paths and barriers of the defects (force convergence criterion: $0.02 \mathrm{~V} \cdot \AA^{-1}$ ).

\section{RESULTS AND DISCUSSION}

Crystal Structures and Simulated STM Images. The energetically favorable crystal structures of the point defects under study are shown in Figure 1. To aid their identification in future experimental works, STM images are simulated at a voltage of $2 \mathrm{~V}$ and included in Figure 1. Results for pristine BlueP are shown for comparison in Figure 1a. We find that pristine BlueP has $\mathrm{C}_{3 \mathrm{v}}$ symmetry after structure relaxation. The optimized lattice constant (3.28 $\AA$ ), buckling height (2.26 $)$, and P-P bond length $(1.24 \AA)$ of pristine BlueP confirm previous results, 11,59 and the triangular patterns in the simulated STM image agree with experiment. $\underline{26,27}$
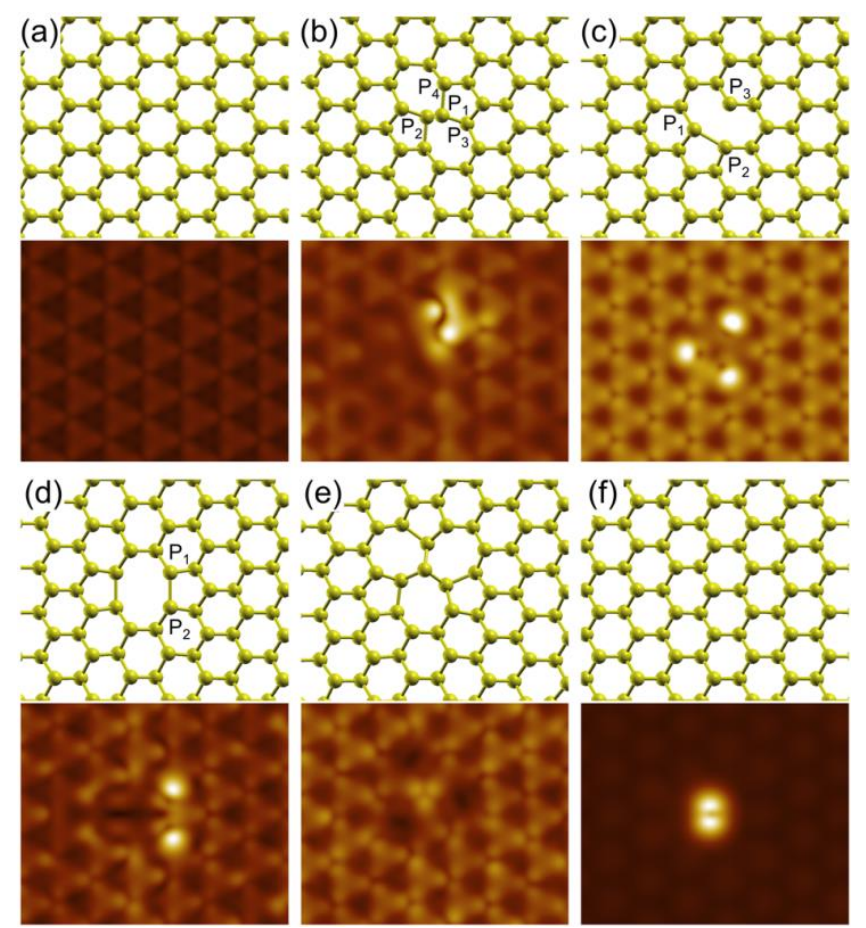

Figure 1. Crystal structures (upper panels) and simulated STM images under a bias of $2 \mathrm{~V}$ (lower panels): (a) pristine BlueP; BlueP with (b) SW (55-77), (c) SV (5-9); (d) DV1 (5-85) and (e) DV2 (555-777) defects; (f) BlueP with P adatom.

We create a SW defect by rotating one P-P bond by a $90^{\circ}$ angle around its center. The optimized structure is shown in Figure $1 \mathrm{~b}$. Instead of parallel alignment, we observe an angle of $24^{\circ}$ between the rotated $\mathrm{P}_{1}-\mathrm{P}_{2}$ bond and the zigzag direction of BlueP. This finite angle causes irregularities in the shapes of the two 5-atomic and two 7-atomic rings of the (55-77) structure, similar to arsenene $\underline{54}$ but different to graphene $\underline{29}$ and silicene. $\underline{44}$ The rotated $\mathrm{P}_{1}-\mathrm{P}_{2}$ bond is shortened to $2.19 \AA$ and the adjacent $\mathrm{P}_{1}-\mathrm{P}_{3}$ and $\mathrm{P}_{1}-\mathrm{P}_{4}$ bonds are elongated to 2.29 and $2.36 \AA$, respectively. The STM image in Figure $1 \mathrm{~b}$ shows two bright spots (at atoms $\mathrm{P}_{1}$ and $\mathrm{P}_{4}$ ) separated by a distance of $2.01 \AA$. The strong asymmetry of the STM image, which is due to the buckling of BlueP, makes it difficult to correlate it with the crystal structure.

The SV defect is obtained by removing one $\mathrm{P}$ atom. We consider the initial structures shown in Figure S1 in the Supporting Information, which all are stable in the case of arsenene. .44 For BlueP we find that the structures of Figures S1b and S1c are not stable. Similar to graphene and silicene, the favorable structure, see Figure 1c, is subject to a Jahn-Teller distortion that saturates the dangling bonds of atoms $\mathrm{P}_{0}$ and $\mathrm{P}_{1}$ to lower the total energy. This 5-9 structure possesses one 5-atomic and one 9-atomic ring. The $\mathrm{P}_{1}$ $\mathrm{P}_{2}$ bond length is $2.64 \AA$, indicating formation of a chemical bond. The SV defect in BlueP is easy to identify, because the STM image in Figure 1c shows three characteristic bright spots forming a triangle.

We find two types of DVs in BlueP: DV1 (5-8-5) with two 5atomic and one 8-atomic rings and DV2 (555-777) with three 5-atomic rings and three 7 -atomic rings, see Figure $1 \mathrm{~d}$ and $1 \mathrm{e}$, respectively. DV1 is obtained by removing one pair of neighboring $\mathrm{P}$ atoms followed by structure relaxation. This DV type is well known from graphene. The $\mathrm{P}_{1}-\mathrm{P}_{2}$ bond length is $2.58 \AA$, i.e., all dangling bonds are saturated. DV2 is derived from DV1 by rotating one P-P followed by structure relaxation. The STM image in Figure 1d shows for DV1 a pair of bright spots (at atoms $\mathrm{P}_{1}$ and $\mathrm{P}_{2}$ ) separated by a distance of $3.66 \AA$. This pattern is symmetric and therefore easy to identify. The STM image of the DV2 defect in Figure 1e is asymmetric, like that of the SW defect, with three relatively bright spots induced by the $\mathrm{P}$ atoms shared by the three 7 -atomic rings.

For the $\mathrm{P}$ adatom we consider the top, valley, bridge, and hollow sites of BlueP, as shown in Figure S2. The top site turns out to be energetically favorable, consistent with a previous prediction. $\underline{6}$ More sprecisely speaking, the $\mathrm{P}$ adatom is slightly shifted off the top site, with a short bond length of $2.06 \AA$, see the optimized structure and simulated STM image in Figure 1f. The $\mathrm{P}$ adatom protrudes from the host to a height of $1.89 \AA$ and leads to a very bright double spot in the STM image.

To assess the stability of the defects, the formation energies are calculated as

$$
E_{\mathrm{f}}=E-N \times E_{\mathrm{P}}
$$

where $E$ is the total energy of defective BlueP, $N$ the number of $\mathrm{P}$ atoms in defective BlueP, and $E_{\mathrm{P}}$ the energy of one $\mathrm{P}$ atom in pristine BlueP. We obtain for the SW, SV, DV1, DV2, and P adatom defects values of 1.60, 2.38, 2.85, 2.32, and $1.97 \mathrm{eV}$, respectively, which are significantly smaller than those of defects in graphene (4.50-9.00 eV) $\underline{29}$ but resemble those of defects in silicene $(-0.03-3.77 \mathrm{eV}), \underline{44}$ black phosphorene (1.01-3.04 eV), $\underline{60}$ and arsenene (1.08-2.17 $\mathrm{eV}) . \underline{54}$ As the SW defect has the lowest formation energy, the probability of its formation is the highest. The SV defect has about $5 \mathrm{eV}$ lower formation energy in BlueP than in graphene $(7.5 \mathrm{eV}), \underline{29}$ while for silicene $(3.01 \mathrm{eV}), \underline{44}$ black phosphorene (1.63 eV), $\underline{60}$ and arsenene (2.17 eV) $\underline{54}$ comparable values have been reported. Similar to graphene, silicene, and arsenene, but not to black phosphorene, in BlueP 
the DV1 defect is less stable than the DV2 defect (by 0.53 $\mathrm{eV}$ ), indicating that it may transform into a DV2 defect by rotation of a P-P bond. The fact that the formation energies of the DV1 and DV2 defects are much lower than twice the formation energy of the SV defect indicates much higher thermodynamical stability, i.e., merging of two SV defects generates one DV defect with high probability.

To further evaluate the defect formation in BlueP at a finite temperature $T$, the areal defect density $n$ is estimated using the Arrhenius equation,

$$
n=n_{0} \exp \left(-E_{\mathrm{f}} / k_{\mathrm{B}} T\right),
$$

where $n_{0}$ is the areal atom density, $E_{f}$ is the formation energy of the defect, and $k_{\mathrm{B}}$ is the Boltzmann constant. Since $n_{0}=2.15 \times 10^{19} \mathrm{~m}^{-2}$, we obtain the results shown in Figure 2. Up to very high temperatures the areal defect densities are not comparable to those achieved by ion or electron irradiation $\frac{33-36}{3}$. At $533 \mathrm{~K}$ (temperature of BlueP synthesis on $\mathrm{Au}(111)$ substrate $\underline{26,27}$ ) all defects have a relatively low areal density, i.e., it is likely that a large high quality BlueP sheet can be obtained by epitaxial growth.

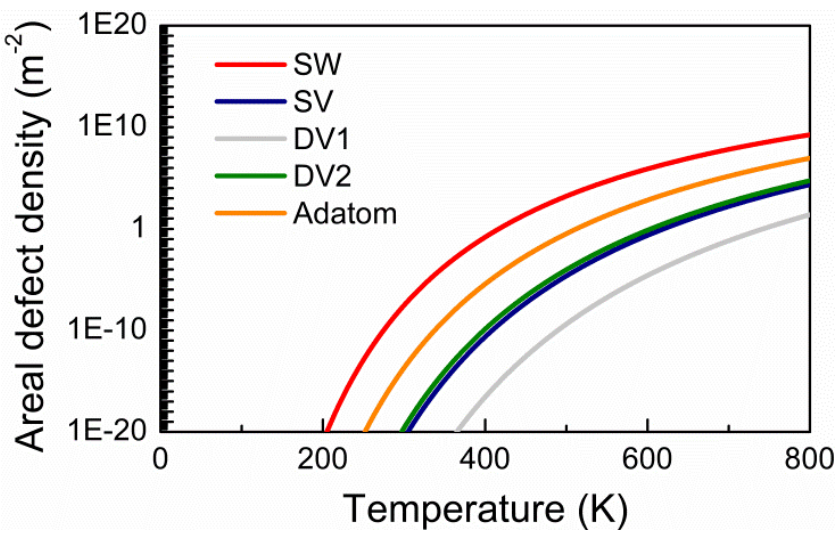

Figure 2. Areal defect densities as functions of temperature.

Thermal stability. The thermal stability of the defects in BlueP is examined by ab-initio molecular dynamics simulations with a time step of $1 \mathrm{fs}$. We first set the temperature to $\mathrm{T}=300 \mathrm{~K}$. After a simulation time of $10 \mathrm{ps}$, Figure 3a-e demonstrates thermal stability at ambient conditions. We then heat up to $500 \mathrm{~K}$ (Figure $3 \mathrm{f}$-j). After a simulation time of 10 ps, the SW defect has disappeared, indicating that it can be readily eliminated by thermal annealing. The SV and DV1 defects show signs of decomposition, in contrast to the DV2 defect and BlueP with $\mathrm{P}$ adatom (both showing decomposition at $1000 \mathrm{~K}$ ). The fluctuations of the temperature and total energy in the simulations are given in Figure S3.

Migration and Transformation. Migration and transformation of defects can have significant effects on the properties of 2D materials. Figure 4 a shows the energy profile for formation of a SW defect in BlueP together with the structures of the initial, transition, and final states. While formation of a SW defect is difficult in graphene due to a high barrier of $9.2 \mathrm{eV}, \underline{61}$ we find for BlueP a value of only $2.43 \mathrm{eV}$. Thus, formation is much more likely in BlueP than in graphene. The barrier of the reverse transformation $(0.83 \mathrm{eV})$ also turns out to be lower than in graphene (5.0 eV), $\underline{29}$ confirming that SW defects in BlueP can be eliminated by thermal annealing. As compared to the other defects under study, the SW defect has the highest probability of formation.

The energy profile in Figure $4 \mathrm{~b}$ shows for the migration of a SV defect in BlueP a diffusion barrier of $0.05 \mathrm{eV}$, which is much lower than in graphene $(1.3 \mathrm{eV}) \cdot \frac{62,63}{\mathrm{It}}$ is similar to that in silicene $(0.12 \mathrm{eV}), \underline{44}$ black phosphorene $(0.18 \mathrm{eV}), \underline{64}$ and arsenene $(0.05 \mathrm{eV}) . \underline{54}$ The low diffusion barrier may permit experimental access to migration dynamics even at room temperature. Due to the high diffusion coefficient, SV defects in BlueP are prone to transforming into DV defects. In the case of migration of a DV1 defect, see Figure 4c, we obtain three transition states (TS1, TS2, and TS3) as well as two metastable states (IM1 and IM2). Initially, the 8atomic ring is deformed by overcoming a barrier of $0.71 \mathrm{eV}$ to reach IM1. Passing through TS2 with a 4-atomic ring, IM2 again shows an 8-atomic ring, however, shifted by one hexagon as compared to IM1, which results in migration of (a)

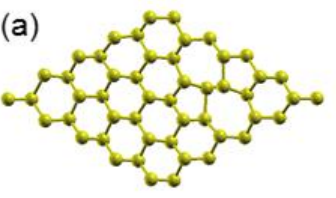

(b)
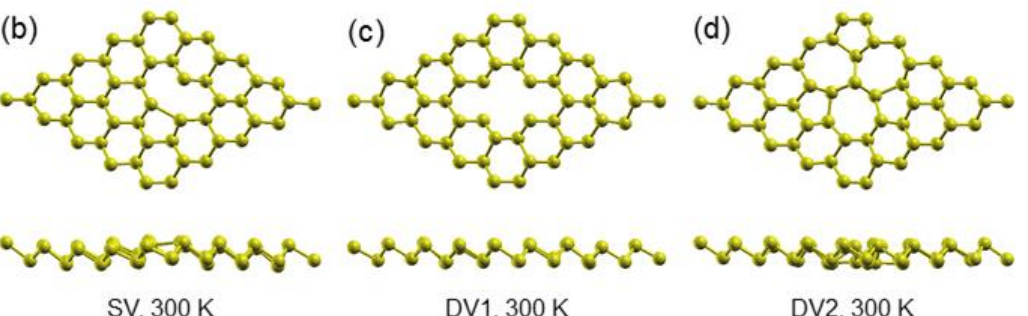

DV $1,300 \mathrm{~K}$

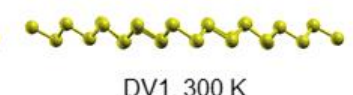

DV2, $300 \mathrm{~K}$
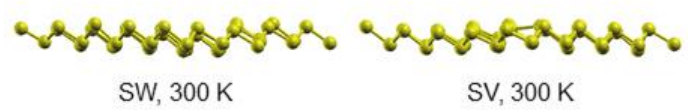
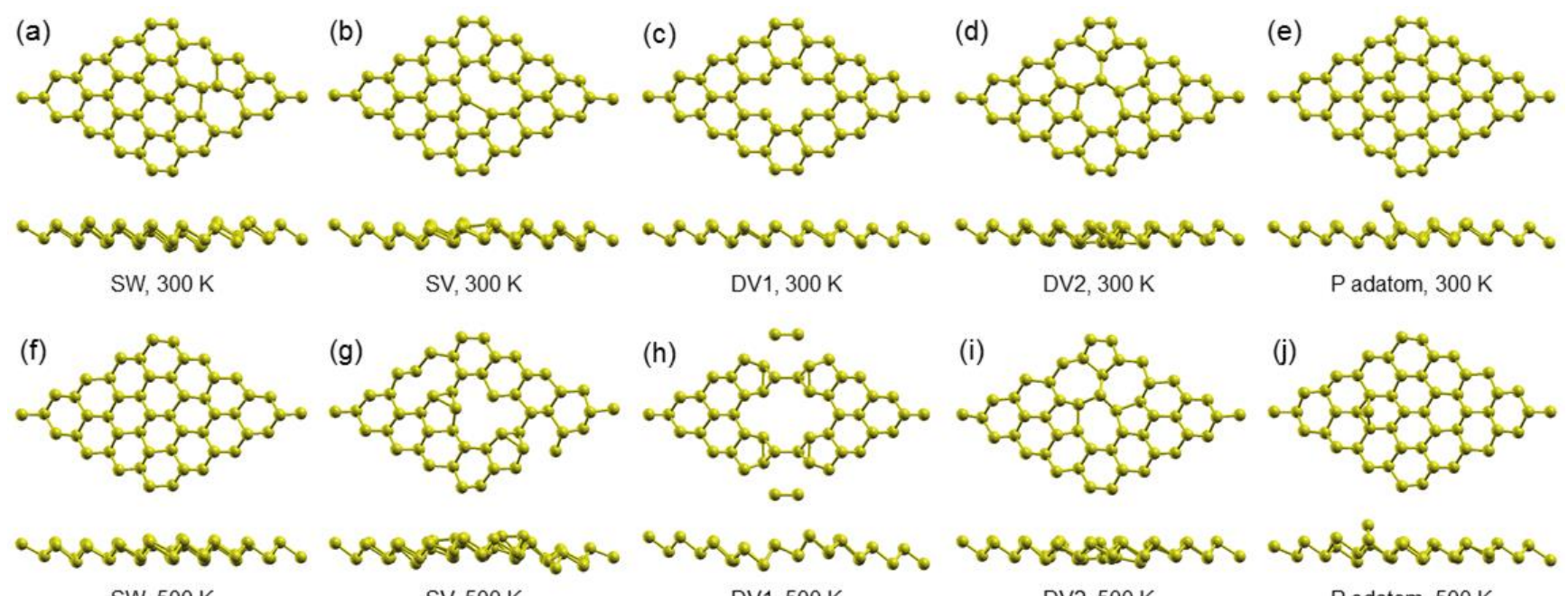

SW, $500 \mathrm{~K}$

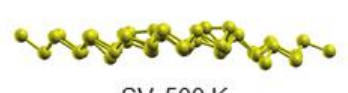

SV, $500 \mathrm{~K}$

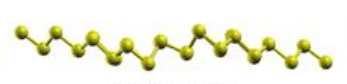

DV $1,500 \mathrm{~K}$

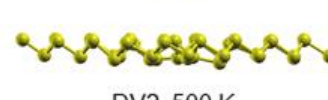

DV2, $500 \mathrm{~K}$
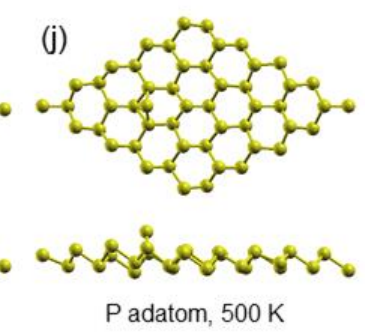

Figure 3. Snapshots at the end of 10 ps ab-initio molecular dynamics simulations at (a-e) $300 \mathrm{~K}$ and (f-j) $500 \mathrm{~K}$ of BlueP with (a, f) SW (55-77), (b, g) SV (5-9), (c, h) DV1 (5-8-5), and (d, i) DV2 (555-777) defects; (e, j) BlueP with P adatom. 

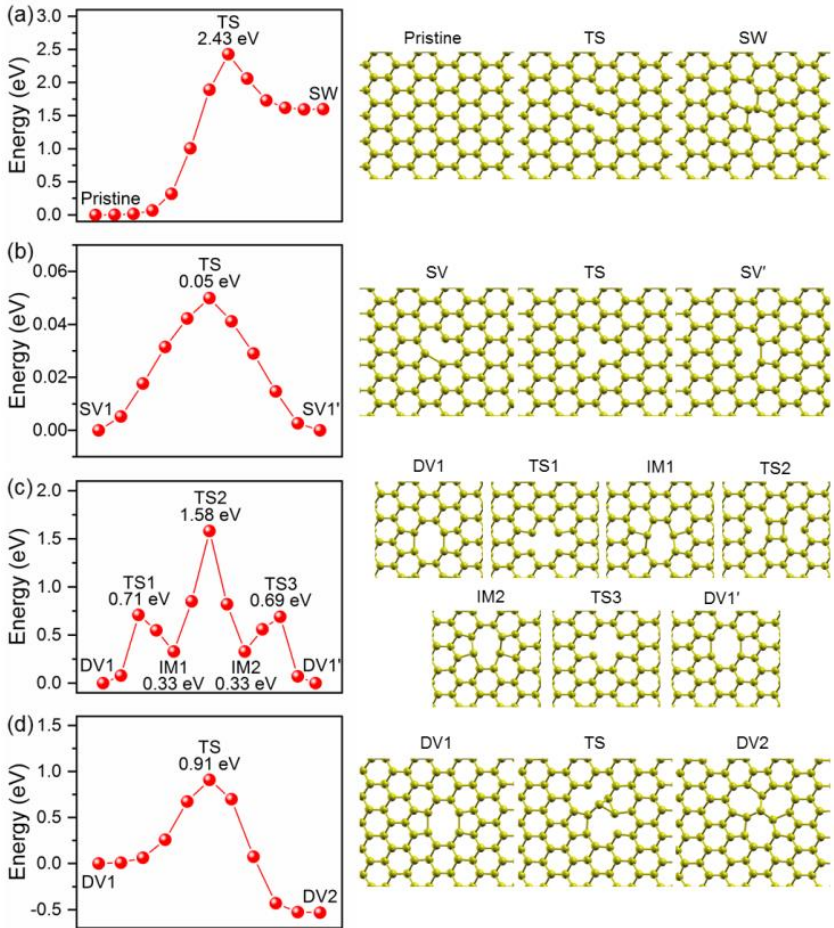

Figure 4. Energy profiles and atomic structures: (a) formation of a SW defect in BlueP, (b) migration of a SV defect, (c) migration of a DV1 defect, and (d) transformation of a DV1 defect into a DV2 defect.

the DV1 defect. In agreement with previous studies of graphene $\underline{29}$ and silicene, $\underline{44}$ it is much more difficult for a DV1 defect to migrate than for a SV defect: The diffusion barrier of $1.58 \mathrm{eV}$ is about 34 times higher than in the case of the SV defect, suppressing migration even at high temperature. Yet, it is low as compared to graphene $(\sim 7 \mathrm{eV}) . \underline{29}$ Transformation of a DV1 defect into a DV2 defect requires rotation of one P-P bond. The energy profile of this process in Figure $4 \mathrm{~d}$ shows that a barrier of $0.91 \mathrm{eV}$ must be overcome, much less than in graphene $(10 \mathrm{eV}) \underline{29}$ and silicene $(2.64 \mathrm{eV}), 44$ indicating that a transformation is realistic.

Electronic, Magnetic, and Optical Properties. Since pristine BlueP is non-magnetic, $\underline{6}$ inducing spin polarization is of vital importance to facilitate application in 2D spintronics. Point defects may serve this purpose. We find that the SV and adatom defects indeed induce spin polarization in BlueP (magnetic moment of $1 \mu_{\mathrm{B}}$ in both cases), whereas the other defects under study have no such effect. This is significant because the spin polarization is carried by $\mathrm{s}$ and p electrons, which supports long-range exchange coupling and a robust magnetic state $\frac{65,66}{}$ The spin density of BlueP with SV defect is shown in Figure 5 a. Since the $\mathrm{P}_{3}$ atom has dangling bonds, it contributes a magnetic moment of 0.58 $\mu_{\mathrm{B}}$. In addition, we find small induced magnetic moments around the defect $\left(0.04,0.04,0.03,0.03,0.06\right.$, and $0.06 \mu_{\mathrm{B}}$ for $\mathrm{P}_{1}, \mathrm{P}_{2}, \mathrm{P}_{4}, \mathrm{P}_{5}, \mathrm{P}_{6}$, and $\mathrm{P}_{7}$, respectively). The spin density of Blue $\mathrm{P}$ with $\mathrm{P}$ adatom is shown in Figure $5 \mathrm{~b}$. While the $\mathrm{p}_{z}$ orbital of the adatom is saturated by bonding with the $\mathrm{P}$ atom below, significant spin density is found in the $s, p_{x}$, and $\mathrm{p}_{y}$ orbitals. The $\mathrm{P}$ adatom carries a magnetic moment of $0.75 \mu_{\mathrm{B}}$, which induces small magnetic moments of 0.06 $\mu_{B}$ on both $\mathrm{P}_{1}$ and $\mathrm{P}_{2}$. Large energy differences between the spin-polarized and spin-degenerated states point to robust magnetic moments in both BlueP with SV defect and BlueP with $\mathrm{P}$ adatom, as required for spintronics materials.

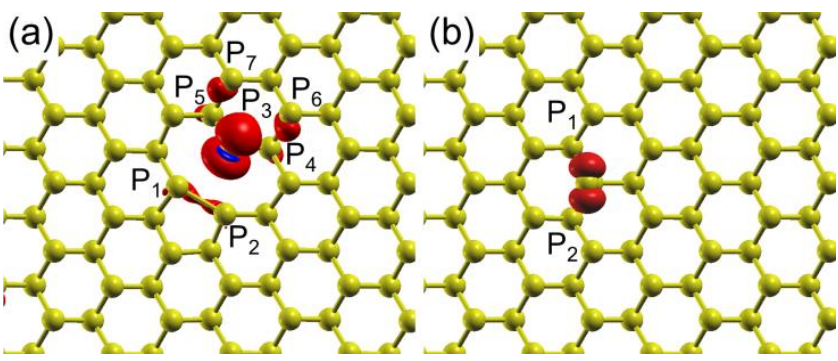

Figure 5. Spin densities of BlueP with (a) SV defect and (b) P adatom. Red and blue isosurfaces refer to the two spin channels. The isosurface value is $0.0125 \mathrm{e} \cdot \AA^{-3}$.

Figure 6 shows the electronic band structures of pristine BlueP and BlueP with defects. Charge densities of selected impurity levels are also given. According to Figure 6a, pristine BlueP is an indirect bandgap semiconductor with the valence band maximum (VBM) located along the line $\Gamma-\mathrm{K}$ and the conduction band minimum (CBM) along the line $\Gamma-$ $\mathrm{M}$. The obtained size of the bandgap $(2.76 \mathrm{eV})$ is consistent with previously reported values. $\underline{\underline{2}}$ The semiconducting behavior is retained after introduction of a SW defect, with a slightly reduced bandgap of $2.57 \mathrm{eV}$ (Figure 6b). An impurity band located just below the CBM reduces the energy required for electronic excitations from the valence to the conduction band, resulting in enhancement of the absorption in the visible and near-infrared spectral ranges, similar to arsenene. $\underline{54}$ The charge density of this level covers not only the SW defect but stretches along the zigzag direction of BlueP, explaining why the impurity band has a dispersion of approximately $0.15 \mathrm{eV}$.

Similar to findings for black phosphorene with SV defect, $\underline{64}$ BlueP with SV defect turns out to be a magnetic semiconductor (Figure 6c), as both spin channels have a bandgap. The charge densities indicate that the in-gap levels mainly belong to the SV defect. BlueP with DV1 defect is a semiconductor with a reduced bandgap of $2.01 \mathrm{eV}$ (Figure 6d). We find an impurity band with a significant dispersion of approximately 0.25 just below the CBM. The charge density of this level is rather delocalized, at least compared to that of the impurity level forming the VBM. Owing to the significant reduction of the bandgap and appearance of the impurity band, enhanced absorption is anticipated.

BlueP with DV2 defect is a semiconductor with a bandgap of $2.41 \mathrm{eV}$ (Figure 6e). Again, we find an impurity band just below the CBM. The dispersion of approximately $0.10 \mathrm{eV}$ is explained by delocalization of the charge density along the zigzag direction of BlueP. On the contrary, the charge density of the impurity level forming the VBM is well localized at the defect. Like BlueP with SV defect, BlueP with P adatom is a magnetic semiconductor (Figure 6f). We observe highly localized in-gap bands in both spin channels. Those of the spin-up channel are occupied $(-0.23$ and $-0.84 \mathrm{eV})$, while in the spin-down channel one is occupied, giving rise to the VBM, and one is empty $(1.20 \mathrm{eV})$. They all are localized at the $\mathrm{P}$ adatom and thus will hardly affect the absorption spectrum. 

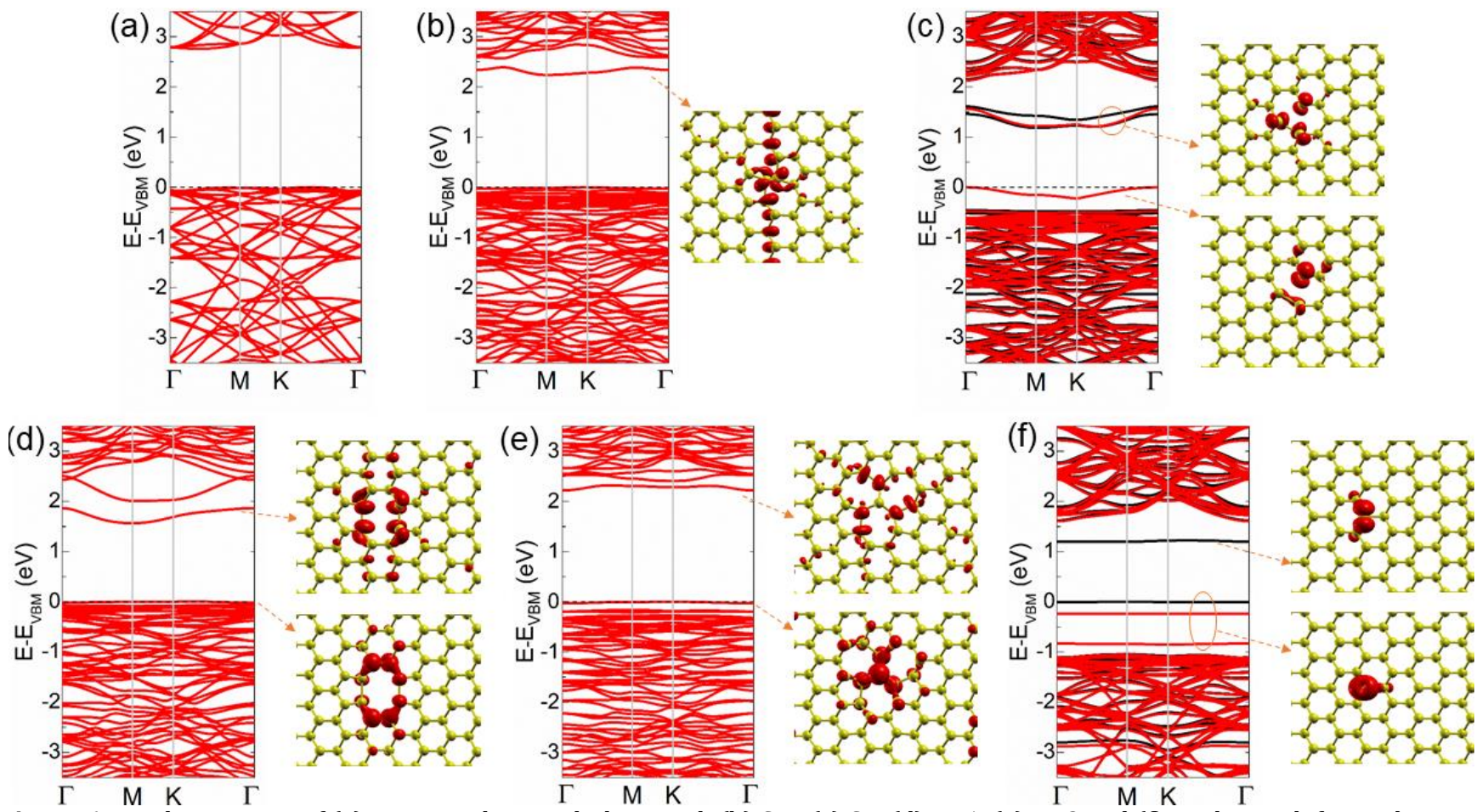

Figure 6. Band structures of (a) pristine BlueP and BlueP with (b) SW, (c) SV, (d) DV1, (e) DV2 and (f) P adatom defects. The VBM is set to zero energy and indicated by a black dashed line. The two spin channels are indicated by red and black solid lines. Charge densities of the impurity levels indicated by arrows are shown with an isovalue of $0.0125 \mathrm{e} \cdot \AA^{-3}$ per level.
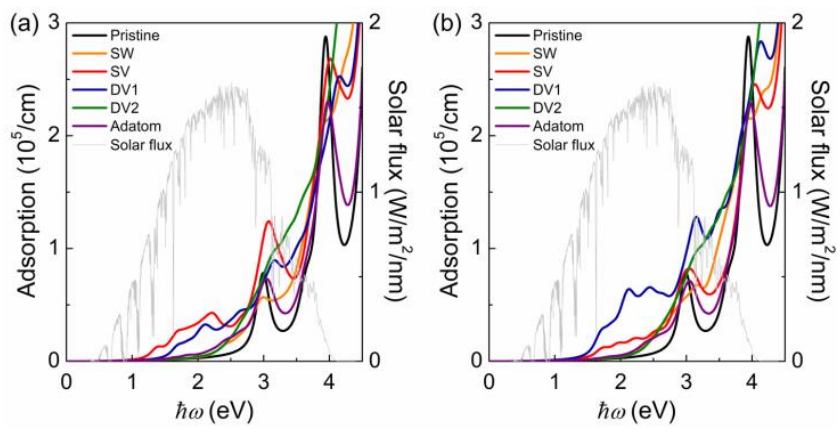

Figure 7. Adsorption spectra of pristine BlueP and BlueP with various defects for polarization along the (a) $x$ and (b) $y$-axis. The incident AM 1.5G solar flux is shown for reference.

We study the optical properties by computing the complex dielectric function, $\varepsilon(\omega)=\varepsilon_{1}(\omega)+\mathrm{i} \varepsilon_{2}(\omega)$, since BlueP has potential as photocatalyst for water splitting. 67 The absorption coefficient is given by

$$
\alpha(\omega)=\frac{\sqrt{2} \omega}{c}\left\{\left[\varepsilon_{1}^{2}(\omega)+\varepsilon_{2}^{2}(\omega)\right]^{1 / 2}-\varepsilon_{1}(\omega)\right\}^{1 / 2} .
$$

Figure 7 depicts the obtained absorption spectra together with the incident AM1.5G solar flux as reference. Pristine BlueP shows the smallest overlap between the absorption spectrum and solar flux regardless of the polarization direction, i.e., the absorption of sunlight is limited. We find that all the defects under consideration significantly affect the absorption spectrum. As compared to pristine BlueP, BlueP with SV defect shows strongly enhanced absorption around $2 \mathrm{eV}$, particularly for polarization along the $x$-axis. The same applies to BlueP with DV1 defect, particularly for polarization along the $y$-axis. For BlueP with DV2 defect we find enhanced absorption, especially above $3 \mathrm{eV}$. We quantify the ability to absorb sunlight by calculating

$$
\eta=\int_{0}^{+\infty} a(\hbar \omega) S(\hbar \omega) \mathrm{d}(\hbar \omega),
$$

where $\hbar \omega, \mathrm{a}(\hbar \omega)$, and $\mathrm{S}(\hbar \omega)$ are the energy, the absorption spectrum, and the incident AM 1.5G solar flux. We obtain for $\eta$ of BlueP with SW, SV, DV1, DV2, and P adatom defects 1.36 (1.55), 2.52 (1.70), 2.12 (3.10), 1.84 (1.81), and 1.37 (1.30) times the value of pristine BlueP, respectively, for polarization along the $x$-axis ( $y$-axis). Thus, the defects significantly enhance the ability of BlueP to absorb sunlight, especially the SV and DV1 defects.

\section{CONCLUSIONS}

We have systemically investigated the structures and STM images of defective BlueP. Defect migration and transformation have been considered, and effects on the electronic, magnetic, and optical properties analyzed. It turns out that the SV defect favors a 5-9 structure, the SW defect an irregular 55-77 structure, and a $\mathrm{P}$ adatom the top adsorption site. The formation energies of the considered defects fall in the range from 1.60 to $2.85 \mathrm{eV}$, i.e., they are significantly lower than in graphene, comparable to silicene, and a bit higher than in arsenene. Due to the lowest formation energy, the SW defect is the most likely defect in BlueP. Evaluation of the areal defect density by the Arrhenius equation 
indicates that high quality BlueP can be obtained by epitaxial growth. Ab-initio molecular dynamics simulations show that the considered defects are thermally stable at ambient conditions. SW defects can be readily eliminated by thermal annealing. In addition, SV and DV1 defects decompose at $500 \mathrm{~K}$, and DV2 and P adatom defects at $1000 \mathrm{~K}$.

Migration and transformation of defects turn out to be easier in BlueP than in graphene. A particularly low diffusion barrier is predicted for the SV defect. Two SV defects can merge into a DV defect, which shows no strong tendency to migration. Transformation of a DV1 defect into a DV2 defect is easier in BlueP than in other 2D materials. SV and P adatom defects induce robust spin-polarization in BlueP, with a magnetic moment of $1 \mu_{\mathrm{B}}$ per defect. All the defects under consideration preserve the semiconducting characteristics of BlueP, implying that the SV and $\mathrm{P}$ adatom defects are interesting for spintronics applications. They also enhance the ability of BlueP to absorb sunlight, which is useful in photocatalysis. Given that there is a lack of experimental work on defects in BlueP, our results provide useful guidelines for future studies.

\section{ASSOCIATED CONTENT}

Supporting Information:

Initial crystal structures of the SV defect; adsorption sites of the $\mathrm{P}$ adatom; fluctuations of the temperature and total energy in the ab-initio molecular dynamics simulations. This material is available free of charge via the internet at http://pubs.acs.org.

\section{AUTHOR INFORMATION}

\section{Corresponding Author}

*U. Schwingenschlögl

E-mail: udo.schwingenschlogl@kaust.edu.sa

\section{ORCID}

Minglei Sun: 0000-0001-5105-0065

Jyh-Pin Chou: 0000-0001-8336-6793

Alice $\mathrm{Hu}$ : 0000-0002-0883-315X

Udo Schwingenschlögl: 0000-0003-4179-7231

\section{Notes}

The authors declare no competing financial interest.

\section{ACKNOWLEDGMENT}

The research reported in this publication was supported by funding from King Abdullah University of Science and Technology (KAUST).

\section{ABBREVIATIONS}

2D, two-dimensional; BlueP, blue phosphorene; CBM, conduction band minimum; DV, double vacancy; STM, scanning tunneling microscopy; SV, single vacancy; SW, Stone-Wales; VBM, valence band maximum.

\section{REFERENCES}

(1) Zhu, Z.; Tománek, D., Semiconducting Layered Blue Phosphorus: A Computational Study. Phys. Rev. Lett. 2014, $112,176802$.
(2) Xiao, J.; Long, M.; Zhang, X.; Ouyang, J.; Xu, H.; Gao, Y., Theoretical Predictions on the Electronic Structure and Charge Carrier Mobility in 2D Phosphorus Sheets. Sci. Rep. 2015, 5, 9961.

(3) Sun, M.; Tang, W.; Ren, Q.; Wang, S.-K.; Yu, J.; Du, Y., A First-Principles Study of Light Non-Metallic Atom Substituted Blue Phosphorene. Appl. Surf. Sci. 2015, 356, 110-114.

(4) Sun, M.; Hao, Y.; Ren, Q.; Zhao, Y.; Du, Y.; Tang, W., Tuning Electronic and Magnetic Properties of Blue Phosphorene by Doping Al, Si, As and Sb Atom: A DFT Calculation. Solid State Commun. 2016, 242, 36-40.

(5) Su, B.; Li, N., Lanthanide Atom Substitutionally Doped Blue Phosphorene: Electronic and Magnetic Behaviors. Phys. Chem. Chem. Phys. 2018, 20, 11003-11012.

(6) Ding, Y.; Wang, Y., Structural, Electronic, and Magnetic Properties of Adatom Adsorptions on Black and Blue Phosphorene: A First-Principles Study. J. Phys. Chem. C 2015, 119, 10610-10622.

(7) Yang, G.; Xu, Z.; Liu, Z.; Jin, S.; Zhang, H.; Ding, Z., Strainand Fluorination-Induced Quantum Spin Hall Insulators in Blue Phosphorene: A First-Principles Study. J. Phys. Chem. C 2017, 121, 12945-12952.

(8) Sun, M.; Wang, S.; Yu, J.; Tang, W., Hydrogenated and Halogenated Blue Phosphorene as Dirac Materials: A First Principles Study. Appl. Surf. Sci. 2017, 392, 46-50.

(9) Xu, W.; Zhao, J.; Xu, H., Adsorption Induced Indirect-toDirect Band Gap Transition in Monolayer Blue Phosphorus. J. Phys. Chem. C 2018, 122, 15792-15798.

(10) Sun, M.; Chou, J.-P.; Yu, J.; Tang, W., Electronic Properties of Blue Phosphorene/Graphene and Blue Phosphorene/Graphene-Like Gallium Nitride Heterostructures. Phys. Chem. Chem. Phys. 2017, 19, 17324-17330.

(11) Du, L.; Zheng, K.; Cui, H.; Wang, Y.; Tao, L.; Chen, X., Novel Electronic Structures and Enhanced Optical Properties of Boron Phosphide/Blue Phosphorene and F4TCNQ/Blue Phosphorene Heterostructures: A DFT + NEGF Study. Phys. Chem. Chem. Phys. 2018, 20, $28777-$ 28785.

(12) Li, J.; Duan, H.; Zeng, B.; Jing, Q.; Cao, B.; Chen, F.; Long, M., Strain-Induced Band Structure Modulation in Hexagonal Boron Phosphide/Blue Phosphorene vdW Heterostructure. J. Phys. Chem. C 2018, 122, 26120-26129.

(13) Pontes, R. B.; Miwa, R. H.; da Silva, A. J. R.; Fazzio, A.; Padilha, J. E., Layer-Dependent Band Alignment of Few Layers of Blue Phosphorus and Their Van Der Waals Heterostructures with Graphene. Phys. Rev. B 2018, 97, 235419.

(14) Peng, L.; Cui, Y.; Sun, L.; Du, J.; Wang, S.; Zhang, S.; Huang, Y., Dipole Controlled Schottky Barrier in the BluePhosphorene-Phase of GeSe Based Van Der Waals Heterostructures. Nanoscale Horiz. 2019 , 4, 480-489.

(15) Li, Y.; Wu, W.; Ma, F., Blue Phosphorene/Graphene Heterostructure as a Promising Anode for Lithium-Ion Batteries: A First-Principles Study with Vibrational Analysis Techniques. J. Mater. Chem. A 2019, 7, 611-620.

(16) Li, J., et al., Electrical Contacts in Monolayer Blue Phosphorene Devices. Nano Res. 2017, 11, 1834-1849.

(17) Li, S.; Wang, T.; Chen, X.; Lu, W.; Xie, Y.; Hu, Y., SelfPowered Photogalvanic Phosphorene Photodetectors with High Polarization Sensitivity and Suppressed Dark Current. 
Nanoscale 2018, 10, 7694-7701.

(18) Bao, J., et al., Hexagonal Boron Nitride/Blue Phosphorene Heterostructure as a Promising Anode Material for Li/Na-Ion Batteries. J. Phys. Chem. C 2018, 122, 23329-23335.

(19) Li, Q.; Yang, J.; Zhang, L., Theoretical Prediction of Blue Phosphorene/Borophene Heterostructure as a Promising Anode Material for Lithium-Ion Batteries. J. Phys. Chem. C 2018, 122, 18294-18303.

(20) Mukherjee, S.; Kavalsky, L.; Chattopadhyay, K.; Singh, C. V., Adsorption and Diffusion of Lithium Polysulfides Over Blue Phosphorene for Li-S Batteries. Nanoscale 2018, 10, 21335-21352.

(21) Mukherjee, S.; Kavalsky, L.; Singh, C. V., Ultrahigh Storage and Fast Diffusion of $\mathrm{Na}$ and $\mathrm{K}$ in Blue Phosphorene Anodes. ACS Appl. Mater. Interface 2018, 10, 8630-8639.

(22) Montes, E.; Schwingenschlögl, U., Superior Selectivity and Sensitivity of Blue Phosphorus Nanotubes in Gas Sensing Applications. J. Mater. Chem. C 2017, 5, 5365-5371. (23) Nanshu, L.; Si, Z., Gas Adsorption on Monolayer Blue Phosphorus: Implications for Environmental Stability and Gas Sensors. Nanotechnology 2017, 28, 175708.

(24) Safari, F.; Moradinasab, M.; Fathipour, M.; Kosina, H., Adsorption of the $\mathrm{NH}_{3}, \mathrm{NO}, \mathrm{NO}_{2}, \mathrm{CO}_{2}$, and $\mathrm{CO}$ Gas Molecules on Blue Phosphorene: A First-Principles Study. Appl. Surf. Sci. 2019, 464, 153-161.

(25) Zhang, J.-J.; Dong, S., Prediction of Above $20 \mathrm{~K}$ Superconductivity of Blue Phosphorus Bilayer with Metal Intercalations. 2D Mater. 2016, 3, 035006.

(26) Zhang, J. L., et al., Epitaxial Growth of Single Layer Blue Phosphorus: A New Phase of Two-Dimensional Phosphorus. Nano Lett. 2016, 16, 4903-4908.

(27) Zhang, W.; Enriquez, H.; Tong, Y.; Bendounan, A.; Kara, A.; Seitsonen, A. P.; Mayne, A. J.; Dujardin, G.; Oughaddou, H., Epitaxial Synthesis of Blue Phosphorene. Small 2018, 14, 1804066.

(28) Lahiri, J.; Lin, Y.; Bozkurt, P.; Oleynik, I. I.; Batzill, M., An Extended Defect in Graphene as a Metallic Wire. Nat. Nanotechnol. 2010, 5, 326-329.

(29) Banhart, F.; Kotakoski, J.; Krasheninnikov, A. V., Structural Defects in Graphene. ACS Nano 2011, 5, 26-41.

(30) Niu, T.; Zhou, M.; Zhang, J.; Feng, Y.; Chen, W., Growth Intermediates for CVD Graphene on $\mathrm{Cu}(111)$ : Carbon Clusters and Defective Graphene. J. Am. Chem. Soc. 2013, 135, 8409-8414.

(31) Zhou, W.; Zou, X.; Najmaei, S.; Liu, Z.; Shi, Y.; Kong, J.; Lou, J.; Ajayan, P. M.; Yakobson, B. I.; Idrobo, J. C., Intrinsic Structural Defects in Monolayer Molybdenum Disulfide. Nano Lett. 2013, 13, 2615-2622.

(32) Jeong, H. Y., et al., Visualizing Point Defects in Transition-Metal Dichalcogenides Using Optical Microscopy. ACS Nano 2016, 10, 770-777.

(33) Compagnini, G.; Giannazzo, F.; Sonde, S.; Raineri, V.; Rimini, E., Ion Irradiation and Defect Formation in Single Layer Graphene. Carbon 2009, 47, 3201-3207.

(34) Lucchese, M. M.; Stavale, F.; Ferreira, E. H. M.; Vilani, C.; Moutinho, M. V. O.; Capaz, R. B.; Achete, C. A.; Jorio, A., Quantifying Ion-Induced Defects and Raman Relaxation Length in Graphene. Carbon 2010, 48, 1592-1597.

(35) Robertson, A. W.; Allen, C. S.; Wu, Y. A.; He, K.; Olivier, J.; Neethling, J.; Kirkland, A. I.; Warner, J. H., Spatial Control of
Defect Creation in Graphene at the Nanoscale. Nat. Commun. 2012, 3, 1144.

(36) Komsa, H.-P.; Kotakoski, J.; Kurasch, S.; Lehtinen, O.; Kaiser, U.; Krasheninnikov, A. V., Two-Dimensional Transition Metal Dichalcogenides Under Electron Irradiation: Defect Production and Doping. Phys. Rev. Lett. 2012, 109, 035503.

(37) Han, W.; Kawakami, R. K.; Gmitra, M.; Fabian, J., Graphene Spintronics. Nat. Nanotechnol. 2014, 9, 794-807.

(38) Amara, H.; Latil, S.; Meunier, V.; Lambin, P.; Charlier, J. C., Scanning Tunneling Microscopy Fingerprints of Point Defects in Graphene: A Theoretical Prediction. Phys. Rev. $B$ 2007, 76, 115423.

(39) Yazyev, 0.; Helm, L., Defect-Induced Magnetism in Graphene. Phys. Rev. B 2007, 75, 125408.

(40) Yazyev, O. V., Emergence of Magnetism in Graphene Materials and Nanostructures. Rep. Prog. Phys. 2010, 73, 056501.

(41) Castro Neto, A. H.; Kotov, V. N.; Nilsson, J.; Pereira, V. M.; Peres, N. M. R.; Uchoa, B., Adatoms in Graphene. Solid State Commun. 2009, 149, 1094-1100.

(42) Wehling, T. O.; Katsnelson, M. I.; Lichtenstein, A. I., Adsorbates on Graphene: Impurity States and Electron Scattering. Chem. Phys. Lett. 2009, 476, 125-134.

(43) Nair, R. R.; Sepioni, M.; Tsai, I. L.; Lehtinen, O.; Keinonen, J.; Krasheninnikov, A. V.; Thomson, T.; Geim, A. K.; Grigorieva, I. V., Spin-Half Paramagnetism in Graphene Induced by Point Defects. Nat. Phys. 2012, 8, 199-202.

(44) Gao, J.; Zhang, J.; Liu, H.; Zhang, Q.; Zhao, J., Structures, Mobilities, Electronic and Magnetic Properties of Point Defects in Silicene. Nanoscale 2013, 5, 9785-92.

(45) Santosh, K. C.; Longo, R. C.; Addou, R.; Wallace, R. M.; Cho, K., Impact of Intrinsic Atomic Defects on the Electronic Structure of $\mathrm{MoS}_{2}$ Monolayers. Nanotechnology 2014, 25, 375703.

(46) Zheng, H.; Yang, B.; Wang, D.; Han, R.; Du, X.; Yan, Y., Tuning Magnetism of Monolayer $\mathrm{MoS}_{2}$ by Doping Vacancy and Applying Strain. Appl. Phys. Lett. 2014, 104, 132403.

(47) Tao, P.; Guo, H.; Yang, T.; Zhang, Z., Strain-Induced Magnetism in $\mathrm{MoS}_{2}$ Monolayer with Defects. J. Appl. Phys. 2014, 115, 054305.

(48) Tang, Y.; Dai, X.; Yang, Z.; Liu, Z.; Pan, L.; Ma, D.; Lu, Z., Tuning the Catalytic Property of Non-Noble Metallic Impurities in Graphene. Carbon 2014, 71, 139-149.

(49) Sun, X.; Liu, Y.; Song, Z.; Li, Y.; Wang, W.; Lin, H.; Wang, L.; Li, Y., Structures, Mobility and Electronic Properties of Point Defects in Arsenene, Antimonene and an Antimony Arsenide Alloy. J. Mater. Chem. C 2017. 5, 4159-4166.

(50) Iordanidou, K.; Kioseoglou, J.; Afanas'ev, V. V.; Stesmans, A.; Houssa, M., Intrinsic Point Defects in Buckled and Puckered Arsenene: A First-Principles Study. Phys. Chem. Chem. Phys. 2017, 19, 9862-9871.

(51) Yuan, S.; Roldán, R.; Katsnelson, M. I.; Guinea, F., Effect of Point Defects on the Optical and Transport Properties of $\mathrm{MoS}_{2}$ and $\mathrm{WS}_{2}$. Phys. Rev. B 2014, 90, 041402.

(52) Chow, P. K.; Jacobs-Gedrim, R. B.; Gao, J.; Lu, T.-M.; Yu, B.; Terrones, H.; Koratkar, N., Defect-Induced Photoluminescence in Monolayer Semiconducting Transition Metal Dichalcogenides. ACS Nano 2015, 9, 15201527.

(53) Liu, X.; Chen, K.-S.; Wells, S. A.; Balla, I.; Zhu, J.; Wood, J. D.; Hersam, M. C., Scanning Probe Nanopatterning and 
Layer-by-Layer Thinning of Black Phosphorus. Adv. Mater. 2016, 29, 1604121.

(54) Liu, X.; Liu, L.; Yang, L.; Wu, X.; Chu, P. K., Optical Identification of Topological Defect Types in Monolayer Arsenene by First-Principles Calculation. J. Phys. Chem. C 2016, 120, 24917-24924.

(55) Kresse, G.; Joubert, D., From Ultrasoft Pseudopotentials to the Projector Augmented-Wave Method. Phys. Rev. B 1999, 59, 1758-1775.

(56) Perdew, J. P.; Burke, K.; Ernzerhof, M., Generalized Gradient Approximation Made Simple. Phys. Rev. Lett. 1996, 77, 3865-3868.

(57) Heyd, J.; Scuseria, G. E.; Ernzerhof, M. Hybrid Functionals Based on a Screened Coulomb Potential. J. Chem. Phys. 2003, 118, 8207-8215.

(58) Henkelman, G.; Uberuaga, B. P.; Jónsson, H., A Climbing Image Nudged Elastic Band Method for Finding Saddle Points and Minimum Energy Paths. J. Chem. Phys. 2000, 113, 9901-9904.

(59) Sun, S.; Hussain, T.; Zhang, W.; Karton, A., Blue Phosphorene Monolayers as Potential Nano Sensors for Volatile Organic Compounds Under Point Defects. Appl. Surf. Sci. 2019, 486, 52-57.

(60) Hu, W.; Yang, J., Defects in Phosphorene. J. Phys. Chem. C 2015, 119, 20474-20480.

(61) Li, L.; Reich, S.; Robertson, J., Defect Energies of
Graphite: Density-Functional Calculations. Phys. Rev. B 2005, 72, 184109.

(62) El-Barbary, A.; Telling, R.; Ewels, C.; Heggie, M.; Briddon, P., Structure and Energetics of the Vacancy in Graphite. Phys. Rev. B 2003, 68, 144107.

(63) Krasheninnikov, A. V.; Lehtinen, P. 0.; Foster, A. S.; Nieminen, R. M., Bending the Rules: Contrasting Vacancy Energetics and Migration in Graphite and Carbon Nanotubes. Chem. Phys. Lett. 2006, 418, 132-136.

(64) Hu, T.; Dong, J., Geometric and Electronic Structures of Mono- and Di-Vacancies in Phosphorene. Nanotechnology 2015, 26, 065705.

(65) Attema, J. J.; de Wijs, G. A.; Blake, G. R.; De Groot, R. A., Anionogenic Ferromagnets. J. Am. Chem. Soc. 2005, 127, $16325-16328$

(66) Kan, E.; Hu, W.; Xiao, C.; Lu, R.; Deng, K.; Yang, J.; Su, H., Half-Metallicity in Organic Single Porous Sheets. J. Am. Chem. Soc. 2012, 134, 5718-5721.

(67) Wang, B.-J.; Li, X.-H.; Zhao, R.; Cai, X.-L.; Yu, W.-Y.; Li, W.-B.; Liu, Z.-S.; Zhang, L.-W.; Ke, S.-H. Electronic Structures and Enhanced Photocatalytic Properties of Blue Phosphorene/BSe Van Der Waals Heterostructures. J. Mater. Chem. A 2018, 6, 8923-8929. 
TOC Graphic

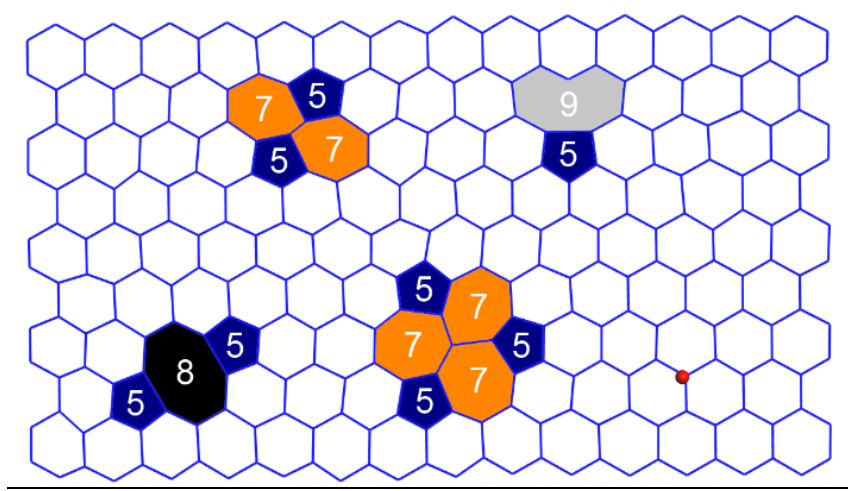

